\title{
Regulation of the length of the fertile period in the domestic fowl by numbers of oviducal spermatozoa, as reflected by those trapped in laid eggs
}

\author{
G. J. Wishart* \\ Agricultural and Food Research Council's Institute of Animal Physiology and Genetics, Roslin, \\ Midlothian EH25 9PS, U.K.
}

\begin{abstract}
Summary. The numbers of spermatozoa trapped in the vitelline membrane of laid eggs were counted after staining with the fluorochrome 2,4-diamidino-2-phenylindole. In a group of 24 hens inseminated with different numbers of spermatozoa to produce different lengths of fertile periods, the numbers of spermatozoa in successive eggs from each hen decreased logarithmically with respect to days following insemination. A relationship could be described between the numbers of spermatozoa per unit area of membrane of an egg and the probability of that egg being fertile. After insemination the number of spermatozoa on successively-laid eggs appears to become reduced until a critical value is reached, after which the hen will lay infertile eggs. By estimating the day on which the critical value was achieved, the actual length of the fertile period could be predicted. It is suggested that the numbers of spermatozoa trapped in the vitelline membrane of laid eggs represent those which surround the ovum at the time of fertilization.
\end{abstract}

\section{Introduction}

A particular feature of avian reproduction is the ability of the hen, following a single mating or artificial insemination, to lay a succession of fertile eggs over a period which may vary from days to weeks, depending on the species (see Lake, 1975). This 'fertile period' is achieved by the prolonged survival of spermatozoa in the oviduct, particularly in utero-vaginal and infundibular 'sperm host glands'. Although present in many species (see Lake, 1975) these glands have been particularly well described in the fowl (e.g. Bobr et al., 1964a) and turkey (e.g. Verma \& Cherms, 1964), in which the efficiency of oviducal sperm storage and resultant fertility are of commercial importance. The relationship between the numbers of spermatozoa held within the oviduct and their rate of decline is, however, unknown. This work describes such a relationship by equating the relative numbers of free infundibular spermatozoa on each day after insemination with numbers of spermatozoa counted in the vitelline membrane of laid eggs, after staining with a DNA-specific fluorochrome.

\section{Materials and Methods}

\begin{abstract}
Birds. Males were from a Rhode Island Red-type control strain and females were a commercial layer strain, both from Ross Poultry Ltd, Newbridge, Midlothian. All birds were caged individually, given $14 \mathrm{~h} \mathrm{light} / 24 \mathrm{~h}$ and fed a commercial breeders ration ad libitum.

Semen collection and artificial insemination. Semen (containing approximately $4 \times 10^{9}$ spermatozoa/ml) was collected as described by Lake (1957) and diluted 2-, 4-, 8- and 16-fold in a glutamate-based diluent (Wishart, 1982). Single intravaginal inseminations of $40 \mu \mathrm{l}$ volumes were made into $4-8$ hens for each of the four dilutions. Eggs were collected from all birds during Days 2 to 21 after insemination and stored at $5^{\circ} \mathrm{C}$.
\end{abstract}

*Present address: Department of Molecular and Life Sciences, Dundee College of Technology, Bell Street, Dundee DD1 1 HG, U.K. 
Counting of spermatozoa in the vitelline membrane. Each egg was opened and assessed for the presence or absence of a developed embryo by the appearance of the germinal disc (Kosin, 1945). A 2-3 cm square of vitelline membrane was then cut and removed from around the germinal disc area. This was rinsed twice, fairly vigorously, in $\mathrm{Ca}^{2+}{ }_{-}$, $\mathrm{Mg}^{2+}$-free Dulbecco's phosphate-buffered saline (PBS) (Dulbecco \& Vogt, 1954), to remove adherent yolk, and then spread on a glass microscope slide. The preparation was then stained at room temperature by adding a few drops of a $1 \mu \mathrm{g} / \mathrm{ml}$ solution of 4,6-diamidino-2-phenylindole in PBS. The entire piece of membrane was then covered with a coverslip, excess liquid was removed and the preparation sealed with nail varnish. When this was dry, two parallel lines, exactly $10 \mathrm{~mm}$ apart, were drawn longitudinally on the coverslip above the membrane, with an indelible marker. The blue fluorescing, comma-shaped sperm nuclei were visualized with a Photomicroscope II (Carl Zeiss, Oberkochen. W. Germany) using an exciting wavelength of $350 \mathrm{~nm}$ and an emission cut-off of $450-500 \mathrm{~nm}$. A defined area of membrane was then assessed for the presence of spermatozoa by scanning perpendicularly between the two marker lines in one movement, focussing up and down to bring into view spermatozoa at different depths in the membrane. Thus a rectangular area $10 \mathrm{~mm} \times 0.549 \mathrm{~mm}$ (the latter number being the diameter of the $\times 25$ objective), i.e. $5.49 \mathrm{~mm}^{2}$ was assessed. Each membrane sample was scanned at different locations 4-6 times.

Treatment of results. All eggs from each hen were scored for fertile status and numbers of spermatozoa on the vitelline membrane, until three infertile eggs were laid consecutively. For each inseminated hen, the length of the fertile period was calculated as the median of the number of days after insemination between the laying of the first infertile and the last fertile egg before three consecutive infertile eggs. Linear regressions were calculated on the logarithm of the numbers of spermatozoa per unit membrane with the number of days after insemination for all eggs laid by each hen. Comparisons were made using Student's $t$ test. Unless otherwise stated, standard errors of the mean are given.

\section{Results}

The appearance of sperm nuclei on the vitelline membrane of the laid egg after staining with diamidinophenylindole is shown in Fig. 1. Their characteristic comma-shape clearly distinguishes them from somatic nuclei (e.g. those of presumed granulosa cells, shown in Fig. 1). Occasionally sperm nuclei were seen in a coiled configuration but were still clearly identifiable.

The numbers of spermatozoa counted per $5.49 \mathrm{~mm}^{2}$ of membrane (subsequently referred to as $\mathrm{N}_{\text {sperm }}$ ) ranged from 0 to 192 , depending on the number of spermatozoa inseminated and the

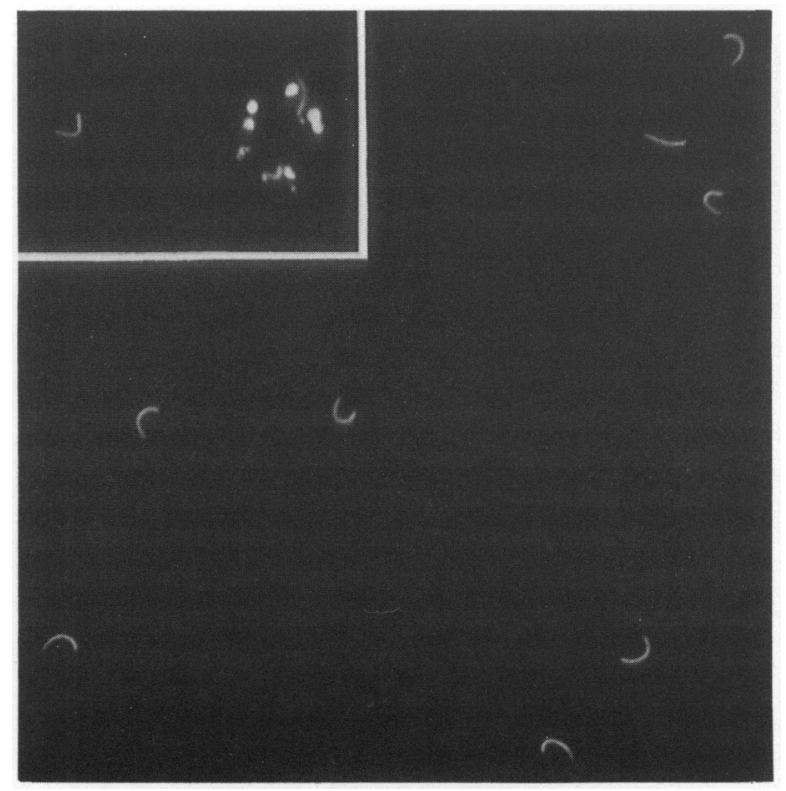

Fig. 1. Fluorescent nuclei of spermatozoa trapped in the vitelline membrane after staining with diamidinophenylindole. Presumed granulosa cell nuclei are also seen. $\times 250$. 


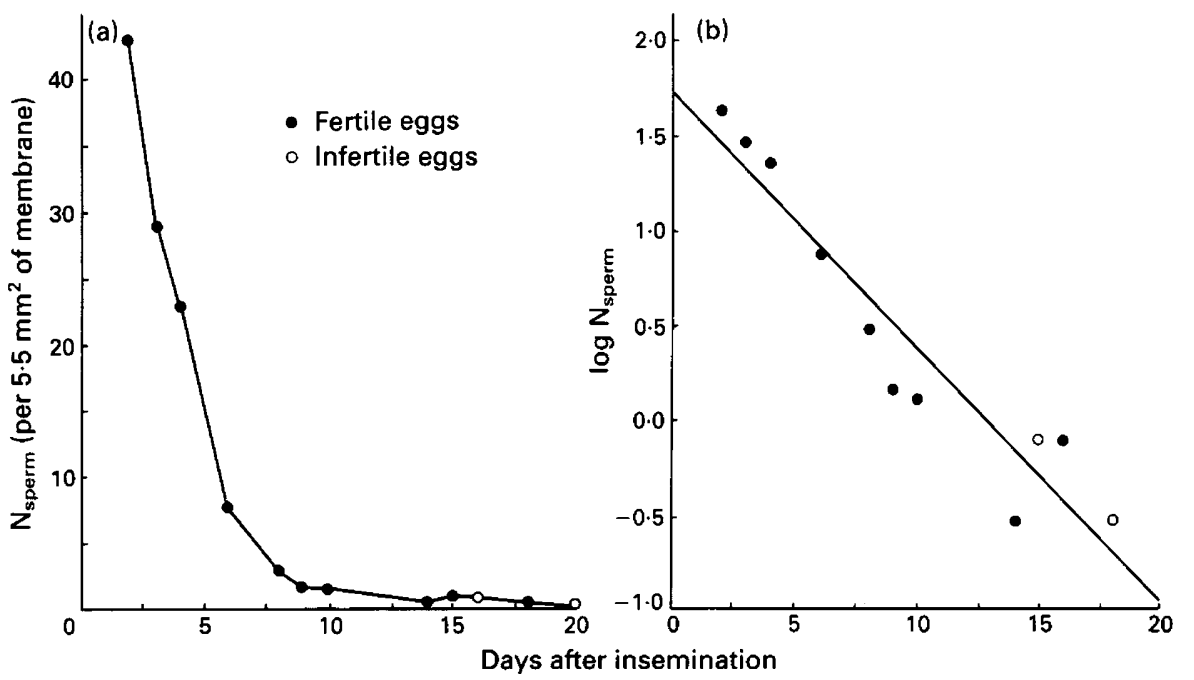

Fig. 2. The decline of $N_{\text {sperm }}$ (a) and $\log \mathrm{N}_{\text {sperm }}$ (b) on the vitelline membrane of eggs laid by one hen after insemination with $60 \times 10^{6}$ spermatozoa.

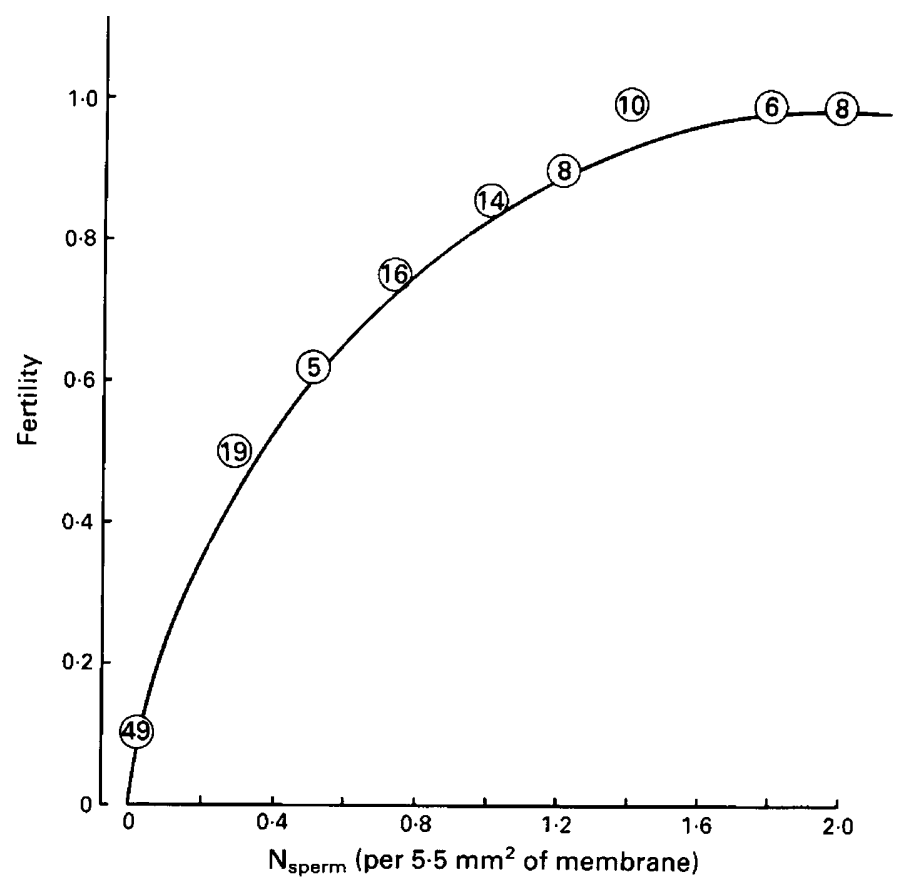

Fig. 3. The relationship between $\mathbf{N}_{\text {sperm }}$ on the vitelline membrane and the probability of fertilization. Eggs from 20 inseminated hens were grouped according to $\mathrm{N}_{\text {sperm }}$ on the vitelline membrane and the fertile status of each group was expressed as a ratio. Inset numbers represent the number of eggs per group. 
number of days between insemination and the laying of the egg from which the membrane was taken. The standard deviations of counts of spermatozoa from 4 different areas of each membrane from a group of 14 eggs ranged from 8.1 to $37.3 \%$ of the mean count, with an average s.d. of $20 \cdot 2 \%$. Although membrane from around the animal pole was routinely taken, pieces from the vegetal pole had similar numbers of entrapped spermatozoa: the mean ratio of animal:vegetal pole counts from 10 eggs at $1.09 \pm 0.14$ was not significantly different from 1.0 with a probability of $5-10 \%$.

The decline of numbers of sperm nuclei in the membrane of eggs laid by one hen after insemination with $60 \times 10^{6}$ spermatozoa is shown in Fig. 2(a). The decrease in numbers, with respect to days after insemination, is approximately logarithmic (Fig. 2b): in a study of 24 hens, with various lengths of fertile periods, the correlation $(r)$ between $\mathrm{N}_{\text {sperm }}$ and days after insemination ranged from $r=0.79$ to 0.98 with an average value of $r=0.92$.

The relationship between the numbers of sperm nuclei counted per $5.49 \mathrm{~mm}^{2}$ of membrane in eggs in which there were fewer than 2 spermatozoa, and the fertile status of these eggs is shown in Fig. 3. For 97 eggs with 2 or more nuclei per unit membrane, only one was infertile. Clearly as $\mathbf{N}_{\text {sperm }}$ decreased to $<1$, the probability of that egg being fertile declined rapidly.

Using the linear regression of $\log \mathrm{N}_{\text {sperm }} v s$ days after insemination, the day on which $\mathrm{N}_{\text {sperm }}$ were $0.4,0.6,0.8$ and 1.0 was determined for all birds for which information was available on 5 or more eggs. When these figures, as predicted lengths of the fertile period, were compared with the actual length of the fertile periods, calculated as described in 'Materials and Methods', correlation coefficients of $0.84,0.89,0.88$ and 0.90 were obtained respectively. The mean values of difference between the actual and predicted fertile periods were $-1.63 \pm 0 \cdot 48,-1 \cdot 18 \pm 0 \cdot 37,+0 \cdot 82 \pm 0 \cdot 29$ and $+1.55 \pm 0.36$ days, respectively. A comparison of the actual length of the fertile period and the predicted $\log \mathrm{N}_{\text {sperm }}$ on the 2nd day after insemination (the day when the first fertile egg was expected), gave a correlation of 0.53 ; with the rate of decline of $\log \mathrm{N}_{\text {sperm }}$ the correlation was -0.27 . Correlations of the logarithm of the number of spermatozoa inseminated with the length of the fertile period, the predicted $\log \mathrm{N}_{\text {sperm }}$ on the 2 nd day after insemination and the rate of decline of $\log \mathrm{N}_{\text {sperm }}$ were $0.61,0.48$ and 0.01 respectively.

\section{Discussion}

The vitelline membrane of the laid hen's egg consists of two main layers, the inner or perivitelline layer and the outer or extravitelline layers, separated by a granular continuous membrane (McNally, 1943; Bellairs et al., 1963). The total width of these layers has been variously reported as between 12 and $24 \mu \mathrm{m}$, discrepancies probably arising from the presence or absence of an outer chalaziferous layer (see Bain \& Hall, 1969). The inner layer is part of the follicular ovum whilst the outer layers are laid down in the infundibulum (Bellairs et al., 1963; Bain \& Hall, 1969). Although Bobr et al. (1964b) observed that spermatozoa appeared to be embedded in both layers of the membrane, Bakst \& Howarth (1977) reported, with pictorial evidence, the presence of spermatozoa between the fibres of the outer layers in the vicinity of the continuous membrane, but not penetrating it. Since normal fertilization takes place in the body cavity or upper infundibulum (Olsen \& Neher, 1948) this must be sequentially followed by a laying down of the outer layers of the vitelline membrane. The numbers of spermatozoa trapped in the initial layers of these membranes are therefore likely to be representative of the numbers which surround the ovum at the time of fertilization. Such an hypothesis would predict a random distribution of spermatozoa within the membrane area, as shown by Bobr et al. (1964b) and confirmed in the present work.

Bobr et al. (1964b) used a haematoxylin-based stain with light microscopy to identify spermatozoa trapped in the vitelline membrane. We have found that spermatozoa stained by this method are less easy to distinguish from background artefacts. The present method has the advantage of the specificity of the fluorochrome for DNA, Bobr et al. (1964b) reported that up to 5000 spermatozoa per egg could be counted on the membrane if inseminations were made into the uterus but 
that only a 'few hundred' were found after intravaginal insemination. Furthermore, they did not find that the numbers of spermatozoa in a series of eggs decreased until the 11 th or 12 th day after insemination, after which numbers fell to zero by the 15 th day. It is possible that the discrepancy between these and the present results reflects different strains of birds used, but it may also reflect the increased resolution of sperm nuclei stained by the present method. Assuming a radius for the average egg to be $15 \mathrm{~mm}$, then the total area of vitelline membrane will be $2829 \mathrm{~mm}^{2}$. The 191 spermatozoa found in $5.49 \mathrm{~mm}^{2}$ of the first fertile egg laid by a bird inseminated with $60 \times 10^{6}$ spermatozoa therefore represents a total of 99129 spermatozoa per membrane, about $0 \cdot 2 \%$ of the inseminated dose.

The mechanism of release of spermatozoa from the sperm-host glands has received considerable attention, with an episodic release coinciding with ovulation or oviposition as an attractive and efficient, although disputed hypothesis (see Bakst, 1981). The present method, being non-invasive and quantitative, shows that 'squeezing' of spermatozoa out of the utero-vaginal glands during oviposition (Grigg, 1957) is not wholly necessary for ensuring a supply of spermatozoa for the nextovulated egg: the numbers of spermatozoa in eggs laid after a non-laying day are not lower than the general logarithmic decline model would predict (see Fig. 2).

The method used for describing the length of the 'actual' fertile period should determine the number of days until an egg with a 50:50 chance of being fertile is laid. From Fig. 3 it would appear that such eggs should have $0 \cdot 3-0 \cdot 4$ spermatozoa per unit membrane, although the predicted fertile period calculated as the number of days until an egg with $\mathrm{N}_{\text {sperm }}=0.4$ is laid gives an overestimation of the actual fertile period. The reasons for this discrepancy remain unknown. It may be that the logarithmic decline model is an oversimplification of the actual pattern but may also reflect the difficulty of obtaining good quantitative information from eggs laid late in the fertile period, when only a few spermatozoa are found in a large area of membrane. It is, however, clear from Fig. 4 that the model described may be used to give a good prediction of the length of the fertile period.

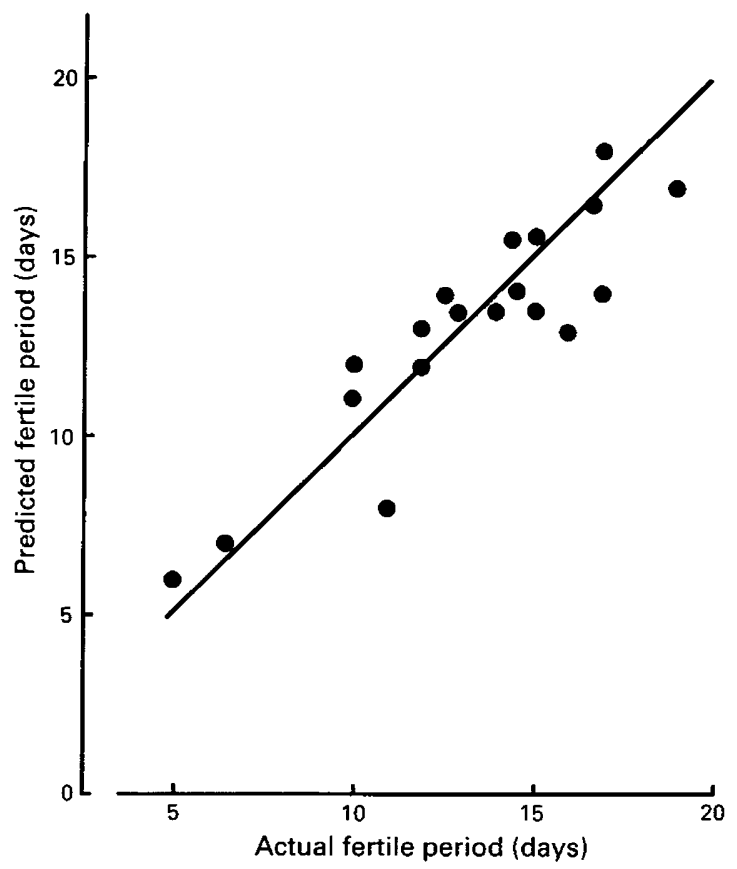

Fig. 4. Correlation of the actual fertile period and that predicted from $\mathbf{N}_{\text {sperm }}$ on the vitelline membrane. Using the logarithmic decline model, the day on which $\mathrm{N}_{\text {sperm }}=0.6$ was calculated for each hen as the last day of the predicted fertile period. A $45^{\circ}$ line is shown for reference. 
These results show that the length of the fertile period in domestic fowls may be considered to be a function of two parameters: (1) the numbers of spermatozoa which are initially accepted by the oviduct after intravaginal insemination and stored in the sperm-host glands and (2) the rate at which these numbers decline or rather are released from the glands. The length of the fertile period appears to be more a function of the former rather than the latter, the rate of decline showing little or no correlation with the length of the fertile period or the numbers of spermatozoa inseminated. The large variation in the numbers of spermatozoa found in the vitelline membrane complex of the first fertile egg from hens inseminated with the same dose of spermatozoa may, therefore, result from differences in the numbers of spermatozoa entering the oviduct or from the capacity of the sperm-host glands.

I thank Miss F. Palmer and Mrs K. Beattie for skilled assistance and Dr P. E. Lake for helpful discussion.

\section{References}

Bain, J.M. \& Hall, J.M. (1969) Observations on the development and structure of the vitelline membrane of the hen's egg: an electron microscope study. Aust. J. biol. Sci. 22, 653-655.

Bakst, M.R. (1981) Sperm recovery from oviducts of turkeys at known intervals after insemination and oviposition. J. Reprod. Fert. 62, 159-164.

Bakst, M.R. \& Howarth, B. (1977) Hydrolysis of the hen's perivitelline layer by cock sperm in vitro. Biol. Reprod. 17, 370-379.

Bellairs, R., Harkness, M. \& Harkness, R.D. (1963) The vitelline membrane of the hen's egg: a chemical and electron microscopical study. J. Ultrastruct. Res. 8, 339-359.

Bobr, L.W., Lorenz, F.W. \& Ogasawara, F.X. (1964a) Distribution of spermatozoa in the oviduct and fertility in domestic birds. I. Residence sites of spermatozoa in fowl oviducts. J. Reprod. Fert. 8, 39-47.

Bobr, L.W., Lorenz, F.W. \& Ogasawara, F.X. (1964b) Distribution of spermatozoa in the oviduct and fertility in domestic birds. II. Transport of spermatozoa in the fowl oviduct. J. Reprod. Fert. 8, 49-58.

Dulbecco, R. \& Vogt, M. (1954) Plaque formation and isolation of pure lines with poliomyelitis viruses. $J$. exp. Med. 97, 169-199.
Grigg, G.W. (1957) The structure of stored sperm in the hen and the nature of the release mechanism. Poult. Sci. 36, 450-451.

Kosin, I.I. (1945) The accuracy of the macroscopic method in identifying fertile unincubated germ discs. Poult. Sci. 24, 281-295.

Lake, P.E. (1957) Fowl semen as collected by the massage method. J. agric. Sci., Camb. 49, 120-126.

Lake, P.E. (1975) Gamete production and the fertile period with particular reference to domesticated birds. Symp. zool. Soc. London. No. 35, 225-244.

McNally, E.H. (1943) The origin and structure of the vitelline membrane of the domestic fowl's egg. Poult. Sci. 36, 40-43.

Olsen, M.W. \& Neher, B.H. (1948) The site of fertilisation in the domestic fowl. J. exp. Zool. 109, 355-366.

Verma, O.P. \& Cherms, F.L. (1964) Observations on the oviduct of turkeys. Avian Diseases 8, 19-26.

Wishart, G.J. (1982) Maintenance of ATP concentrations in and fertilizing ability of fowl and turkey spermatozoa in vitro. J. Reprod. Fert. 66, 457-462.

Received 13 October 1986 